\title{
NMR Probing Spin Excitations in the Ring-Like Structure of a Two-Subband System
}

\author{
X. C. Zhang* G. Scott, and H. W. Jiang \\ Department of Physics and Astronomy, University of California at Los Angeles, \\ 405 Hilgard Avenue, Los Angeles, CA 90095, USA
}

(Dated: August 10, 2018)

\begin{abstract}
Resistively detected nuclear magnetic resonance (NMR) is observed inside the ring-like structure, with a quantized Hall conductance of $6 e^{2} / h$, in the phase diagram of a two subband electron system. The NMR signal persists up to $400 \mathrm{mK}$ and is absent in other states with the same quantized Hall conductance. The nuclear spin-lattice relaxation time, $T_{1}$, is found to decrease rapidly towards the ring center. These observations are consistent with the assertion of the ring-like region being a ferromagnetic state that is accompanied by collective spin excitations.

PACS numbers: 73.43.Nq, 71.30.+h, 72.20.My
\end{abstract}

A two-dimensional electron system consisting of two filled subbands is emerging as an experimental laboratory to study charge and spin correlation effects. The correlations become particularly prominent when the two sets of Landau levels with different subband quantum numbers are brought into degeneracy by varying magnetic field and/or carrier concentration. A series of experimental observations were made to explore the consequences of real electron spin exchange interactions [1, 2], as well as pseudospin charge excitations in the vicinity of the degeneracy regions [3, 4].

One of the interesting findings in the recent studies of the two-subband system is that the experimental phase diagram, in the density-magnetic field plane, exhibits pronounced "ring-like structures" at even integer filling factors [1]. It was conjectured that these structures represent ferromagnetic phases. This conjecture was based on theoretical predictions that a ferromagnetic phase transition can occur when two Landau levels (LLs) with opposite spin are close to crossing at the Fermi energy. By promoting all the electrons, or holes, in the uppermost occupied LL to the lowest unoccupied LL of opposite spin, the total energy of the system can be lowered by the exchange mechanism [5]. Such a transition has been seen in a single subband two dimensional hole system when two LL levels with opposite spin and different Landau level index approach each other by increasing the Zeeman energy using a tilted magnetic field [6]. The ring-like structures have also been observed recently by Ellenberger et al. on a two-subband parabolic quantum well 2]. The authors, however, suggest that the ring structures can be single particle states with an enhanced exchange interaction within each subband in the framework of mean-field theory.

To address the question whether the ring structures are collective states in nature, measurements other than the conventional transport are needed. The resistively detected NMR technique has recently emerged as an effective method to probe collective spin states in the fractional quantum Hall regime [7, 8], the Skyrmion spin texture close to the filling factor $\nu=1$ [9, [10], and the role of electron spin polarization in the phase transition of a bilayer system 11]. This method is significantly less demanding than the high-sensitivity conventional NMR detection [12. In this paper we have adapted this technique to study NMR in the vicinity of the ring structure with a quantized Hall conductance of $6 e^{2} / h$. It reveals that the NMR signal is phase-space dependent and only appears inside the ring structure. An array of observations suggest that the ring-like region is a collective state that has intriguing spin excitations.

The sample used in this study is a symmetrical modulation-doped single quantum well. Both well and spacer are $240 \AA$ thick. The total electron density at gate voltage $V_{g}=0$ is $8.1 \times 10^{11} \mathrm{~cm}^{-2}$, with a distribution of 5.4 and $2.7 \times 10^{11} \mathrm{~cm}^{-2}$ in the first and second subband, respectively. The mobility is $4.1 \times 10^{5} \mathrm{~cm}^{2} / \mathrm{V} \cdot \mathrm{s}$. A NiCr top gated $100 \mu \mathrm{m}$ wide Hall bar with $270 \mu \mathrm{m}$ between voltage probes was patterned by standard lithography techniques. As shown in the inset picture of Fig. 1] several turns of NMR coil were wound around the sample, which was placed in a dilution refrigerator with a base temperature of $60 \mathrm{mK}$, and in a perpendicular magnetic field normal to the sample plane. A small RF magnetic field generated by the coil with a matching frequency $f=\gamma H_{0}$ will cause NMR for ${ }^{75}$ As nuclei, where the gyromagnetic ratio $\gamma=7.29 \mathrm{MHz} / \mathrm{T}$. The resistance was measured using quasi-dc lock-in techniques with $I=100$ $\mathrm{nA}$ and $f=13.2 \mathrm{~Hz}$.

To calibrate the external magnetic field resistively detected NMR close to $\nu=1$ was performed, as shown in Fig. 11 The NMR manifests itself as a dip in the resistance versus frequency curve due to the increased electron Zeeman energy caused by depolarizing the nuclei at resonant conditions [9], see discussions below. The main dip at $33.163 \mathrm{MHz}$ is about $100 \mathrm{KHz}$ separated with two side ones, which are caused by quadrupole splitting resultant from the interaction of the nuclear electric quadrupole moment with an electric field gradient. Here, the splitting is about three times larger than in Ref. [9], possibly due to the large gate voltage applied across the quantum well. By comparing the frequency of the center peak with its theoretical value, the uncertainty of the magnetic field was determined within $\pm 0.1 \%$. 


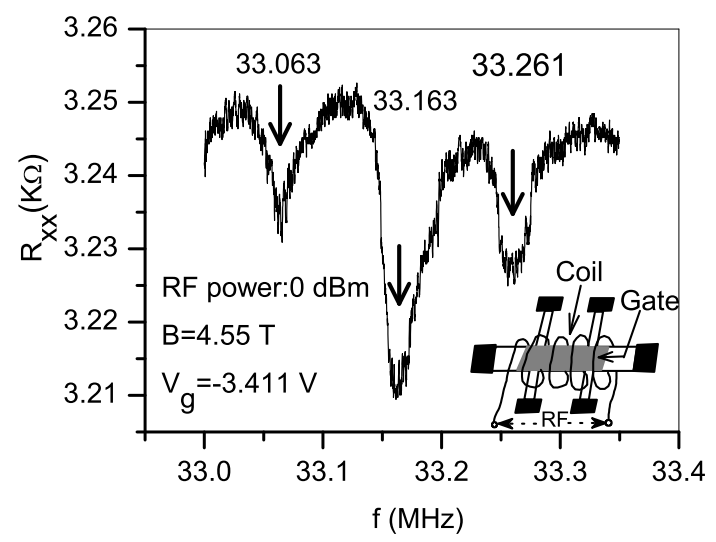

FIG. 1: ${ }^{75}$ As NMR signal detected at $B=4.55 \mathrm{~T}$ and $V_{g}=$ $-3.411 \mathrm{~V}$, close to filling factor $\nu=1$ and in a regime of a single occupied subband. Resonance frequencies are labeled. The center resonance is accompanied by two side quadrupole split dips. The inset shows the gated Hallbar sample around which several turns of coil were wound for passing RF signal.

Fig. 2a shows the phase diagram of longitudinal resistivity, $\rho_{x x}$, as a function of magnetic field, $B$, and carrier density, $n$, at $70 \mathrm{mK}$. Its most salient feature is the ringlike structure centered around the $\nu=6$ line. It was previously speculated that the ring represents a ferromagnetic state realized by preferential alignment of electron spins of different subbands, when separation between LLs of different subbands is comparable with the exchange in-


model is illustrated in Fig. 2b. On either side of the ring the system is in a paramagnetic state with exactly the same quantized Hall conductance, $6 e^{2} / h$, because the topmost two occupied LLs are two spin split states of the same subband. Recently the ring-like structures have been numerically simulated by Ferreira et al. [13].

To probe the aforementioned regions with a means other than transport, resistively detected NMR was performed in the proximity of the ring structure. No NMR was seen on either side of the ring structure, as shown in Fig. 2c and 2e. In contrast, inside the ring structure pronounced NMR signals were observed. An example is shown at $B=4.55 \mathrm{~T}$ for four different values of $V_{g}$. The relative change of $R_{x x}$ is typically about $1 \%$ at resonance at an estimated RF power about $0.1 \mathrm{~mW}$ at the sample. The resonant frequencies at different $V_{g}$ agree well with the theoretical values, indicated by the vertical dashed line in Fig. 2d. Upon resonance, $R_{x x}$ in all NMR lines shows a sharp decrease followed by a much slower relaxation process back to its original value, which is characterized by the nuclear spin-lattice relaxation time constant, $T_{1}$, as will be discussed.

To further confirm the NMR signal, NMR resonance lines were recorded at different magnetic fields inside the
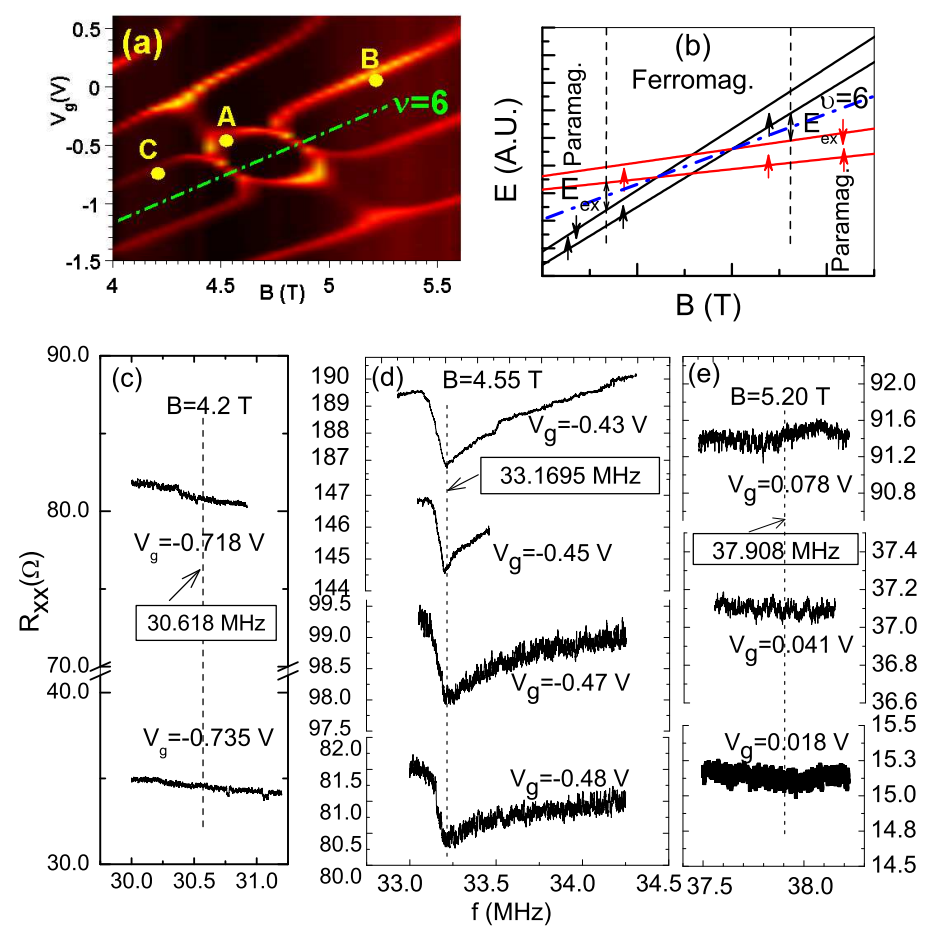

FIG. 2: Fig. (a), Grey scale plot of resistivity versus magnetic field and carrier concentration. Fig. (b), Model of the ferromagnetic phase transition caused by different subband LL spin alignment. Arrows denote spin orientations of LLs close to $\nu=6$ expressed by dot-dashed line. Fig. (c), (d), (e) show NMR signals close to various positions dotted in Fig. (a). Notice that NMR is only detected inside ring structure (Fig. d), and absent on its left (Fig. c) and right side (Fig. e). NMR at a particular $B$ was measured for several different values of $V_{g}$. For a comparison, theoretical NMR values, assuming $\gamma=7.29 \mathrm{MHz} / \mathrm{T}$ for ${ }^{75} \mathrm{As}$, are shown by dashed vertical lines.

ring structure, as shown in Fig. 3k. The resonance shows the expected blue shift with increasing $B$. The frequencies of the minima in the NMR lines, plotted in the inset, exhibit a linear relationship with $B$, and are slightly above the theoretical values denoted by the line. Nevertheless, Fig. 3b reveals that the NMR line form is strongly dependent on frequency sweeping direction and speed. The slower the frequency sweeps, the closer the minima frequency approaches the expected theoretical value. Resistively detected NMR towards the ring center is hampered by the exponentially vanishing $R_{x x}$ value, similar to the earlier study of the Skyrmion state around $\nu=1$ [10].

We believe the mechanism of resistively detected NMR here is similar to that described in the literature. For the $2 \mathrm{D}$ electron system in GaAs, the contact hyperfine interaction between the nuclear spin $\boldsymbol{I}$ and the electron spin $\boldsymbol{S}$ can be expressed as $\boldsymbol{A I} \cdot \boldsymbol{S}=\frac{A}{2}\left(I^{+} S^{-}+I^{-} S^{+}\right)+A I_{z} S_{z}$, where $A$ is the hyperfine coupling constant [15]. Due to the term $\frac{A}{2}\left(I^{+} S^{-}+I^{-} S^{+}\right)$, a nuclear spin flops, $\downarrow \Rightarrow \uparrow$, 
when an electron spin flips, $\uparrow \Rightarrow \downarrow$. Nuclear spins that have once flopped hardly relax back because of their longer relaxation time $T_{1}$, which is on the order of minutes, relative to that of the electrons. Hence up-spin nuclei pile up to develop a strong nuclear polarization $I_{z}$, which is parallel to an external magnetic field $B$. It will reduce the effective electron spin flip energy,

$$
E_{z}=g^{*} \mu_{B} B S_{z}+A\left\langle I_{z}\right\rangle S_{z},
$$

as $g^{*}<0[9]$. When the NMR resonance condition is matched, the nuclear spins are depolarized and the electron Zeeman energy increases consequently. Since $R_{x x}$ is dependent on the energy gap of the energy spectrum, $\Delta E$, and thermally activated according to $R_{x x} \propto$ $\exp (-\Delta E / 2 k T)$, the NMR is manifested by a drop in $R_{x x}$, as shown by all the NMR lines in Fig. 1-3.

The observed prominent (absent) NMR signal inside (outside) the ring structure in Fig. 2c-e, is well correlated with the spin polarization of the picture depicted in Fig. $2 \mathrm{~b}[1]$. Inside the ring along the $\nu=6$ line, the topmost two occupied LLs from different subbands have the same spin orientation and hence $S_{z}=1$ in Eq. (1). Accordingly, NMR signal was observed in Fig. 2d. Whereas outside the ring along the $\nu=6$ line, it is a normal paramagnetic state where spin split LLs of different subbands are completely filled. Hence $S_{z}=0$ in Eq. (1), and NMR cannot be expected.

To gain more insight into the nature of the state inside the ring, we studied the coupling between the nuclei and electrons by measuring the nuclear spin-lattice relaxation time, $T_{1}$, at various positions inside the ring structure. Fig. 4a shows the procedure for determining $T_{1}$ by imitating the nuclear polarization regaining process after complete depolarization. Initially the sample is irradiated by an off-resonance RF. At I, RF was tuned into resonance, and $R_{x x}$ decays exponentially into a steady state, which means that nuclei have been completely depolarized. Then at II, the frequency was switched back to off-resonance, nuclei gradually restore their polarization owing to the interaction with the electron spin bath, and consequently $R_{x x}$ slowly relaxes back to its original value. This relaxing process can be well fitted by an exponential function of the form $R_{x x}=\alpha+\beta e^{\left(-t / T_{1}^{\prime}\right)}$ with a time constant of $T_{1}^{\prime}$, which is approximately equal to the nuclear spin-lattice relaxation time $T_{1}$ if $g \mu_{B} B_{N} \ll 2 k_{B} T$ and the RF power is small 10].

The obtained $T_{1}$ is plotted in Fig. $4 \mathrm{~b}$ as a function of $B$ for different locations inside the ring structure marked by dots in the inset picture. These positions, with their successively diminishing resistance, represent a path progressively leading to the center of the ring structure. Each data point is an average of several repeated measurements. $T_{1}$ rapidly drops from 450 to $95 \mathrm{~s}$ towards the ring center, indicating a more efficient nuclear spin relaxation. Following $R_{x x}$, we found that $T_{1}$ reduces as electrons become more localized. This correlation of $T_{1}$
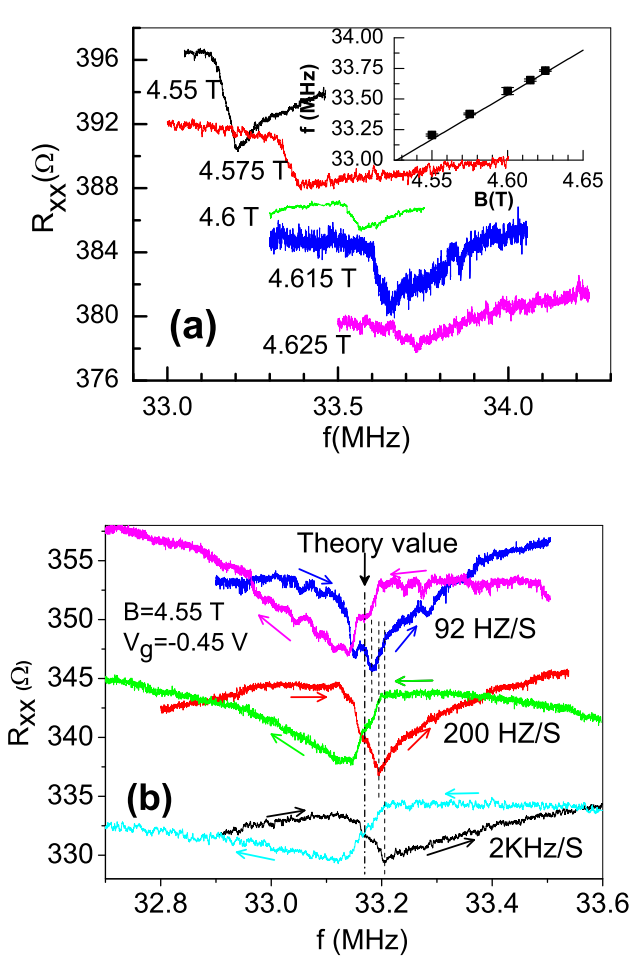

FIG. 3: Fig. (a), NMR resonances at different magnetic fields inside the ring structure. Inset shows the resonance frequency as a function of magnetic field. The straight line has a slope of $7.29 \mathrm{MHz} / \mathrm{T}$ for ${ }^{75}$ As resonance. Fig. (b), detected NMR signals at $B=4.55 \mathrm{~T}$ and $V_{g}=-0.45 \mathrm{~V}$, at three frequency scanning speeds, and in opposite sweeping direction (indicated by arrows). The theoretical resonance frequency at $33.1695 \mathrm{MHz}$ is indicated by dot-dashed vertical line. The curves in both figures are shifted along y-axis for clarity.

with localization is opposite to the usual Korringa relation in metals, $1 / T_{1} \propto D(E) \propto \rho_{x x}, D(E)$ is density of states. However, the data share a remarkable resemblance with that observed by Gervais et al. 10]. In that study, the fast relaxation rate was attributed to the localized Skyrmion crystal relaxing the nuclear spin via a noncollinear spin wave Goldstone mode [14]. Stimulated by this assertion, we intend to suggest that the electrons inside the ring form a many-body state with collective spin excitations. Therefore, the localized electrons can provide an efficient relaxation path for nuclear spins. The theoretically predicted spin-density-wave instabilities near the paramagnetic-ferromagnetic transition [5] can potentially be a source of these quantum fluctuations.

We also found that measurements up to $400 \mathrm{mK}$ with the same frequency sweeping speed reveal negligible change in the line shape of the NMR signal. Assuming a Boltzmann distribution, at $T=400 \mathrm{mK}$ and $B=4.55$ $\mathrm{T}$, the percentage of polarized ${ }^{75} A s, \eta$, is only $0.2 \%$. The corresponding resonance amplitude $\Delta R_{x x} / R_{x x}=$ 



FIG. 4: Fig. (a) shows the principle of measuring $T_{1}$ by recording time evolution of $R_{x x}$ irradiated by RF, initially off resonance, on resonance (position I), and finally off resonance (position II). The on-resonance frequency was set to 33.1695 $\mathrm{MHz} . T_{1}$ is determined by an exponential fit to the experimental data shown by the thick gray curve. Fig. (b), acquired $T_{1}$ for points shown in its inset exhibits a declining tendency towards the center of the ring structure. Inset is a zoom-in view of the ring structure. Dots $\mathrm{A}, \mathrm{B}, \mathrm{C}$, and $\mathrm{D}$ show a path leading towards the ring center, $\mathrm{A}(4.55 \mathrm{~T},-0.45 \mathrm{~V}), \mathrm{B}(4.575$ $\mathrm{T},-0.459 \mathrm{~V}), \mathrm{C}(4.6 \mathrm{~T},-0.468 \mathrm{~V}), \mathrm{D}(4.615 \mathrm{~T},-0.475 \mathrm{~V})$.

$\Delta E_{z} / 2 k T$ is about $0.2 \%$, where $\Delta E_{z}=g^{*} \mu_{B} B_{N}$, and the nuclear field generated by the polarized ${ }^{75} \mathrm{As}$ nuclei $B_{N}=-1.84\left\langle I^{75} A s\right\rangle=-1.84 \cdot(3 / 2 \cdot \eta)$ in Tesla 15 . This is nearly one order of magnitude lower than the experimental value of $1 \%$. Thus the observed nuclear spin polarization cannot be built thermally and it has to be gained by some dynamic means.

Indeed, it was found that electrical current can efficiently induce electron spin-flip and nuclear spin-flop processes in the $\nu=2 / 3$ fractional quantum Hall spinpolarized state [16, 17]. Since a ferromagnetic state can spontaneously separate into domains, as the applied current forces electrons to scatter between adjacent domains with different spin but almost degenerate energy, the nuclei in the neighborhood can become polarized. Adapting this picture of dynamic polarization, we can imagine that strong nuclear polarization observed at high temperatures in our experiment is consistent with the fact that the ring state consists of spontaneously polarized electron spins.
In conclusion, resistively detected NMR was detected only inside the ring-like structure in the phase diagram of a two-subband electron system. The associated nuclear spin-lattice relaxation time decays rapidly towards the center of the ring and the NMR signal persists to temperatures as high as $400 \mathrm{mK}$. These findings are consistent with the notion that there is a ferromagnetic state inside the ring structure facilitated by preferential alignment of electron spins from different subband in an external magnetic field.

The authors would like to thank G. Clark for helpful discussions, and B. Alavi for technical assistance. This work is supported by NSF under grant DMR-0404445.

* Electronic address: xczhang@physics.ucla.edu

[1] X. C. Zhang, D. R. Faulhaber, and H. W. Jiang, Phys. Rev. Lett. 95, 216801 (2005).

[2] C. Ellenberger, B. Simovic, R. Leturcq, T. Ihn, S. E. Ulloa, K. Ensslin, D. C. Driscoll, and A. C. Gossard, cond-mat/0602271

[3] K. Muraki, T. Saku, and Y. Hirayama, Phys. Rev. Lett. 87, 196801 (2001).

[4] X. C. Zhang, I. Martin, and H. W. Jiang, Phys. Rev. B 74, 073301 (2006)

[5] G. F. Giuliani, and J. J. Quinn, Phys. Rev. B. 31, 6228 (1985); Surf. Sci. 170, 316(1986).

[6] A. J. Daneshvar, C. J. B. Ford, M. Y. Simmons, A. V. Khaetskii, A. R. Hamilton, M. Pepper, and D. A. Ritchie, Phys. Rev. Lett. 79, 4449 (1997).

[7] J. H. Smet, R. A. Deutschmann, F. Ertl, W. Wegscheider, G. Abstreiter, and K. von Klitzing, Nature (London) 415, 281 (2002).

[8] O. Stern, N. Freytag, A. Fay, W. Dietsche, J. H. Smet, K. von Klitzing, D. Schuh, and W. Wegscheider, Phys. Rev. B 70, 75318 (2004).

[9] W. Desrat, D. K. Maude, M. Potemski, J. C. Portal, Z. R. Wasilewski, and G. Hill, Phys. Rev. Lett. 88, 256807 (2002).

[10] G. Gervais, H. L. Stormer, D. C. Tsui, P. L. Kuhns, W. G. Moulton, A. P. Reyes, L. N. Pfeiffer, K. W. Baldwin, and K. W. West, Phys. Rev. Lett. 94, 196803 (2005).

[11] I. B. Spielman, L. A. Tracy, J. P. Eisenstein, L. N. Pfeiffer, and K. W. West, Phys. Rev. Lett. 94, 76803 (2005).

[12] S. E. Barrett, G. Dabbagh, L. N. Pfeiffer, K. W. West, and R. Tycko, Phys. Rev. Lett. 74, 5112 (1995); R. Tycko, S. E. Barret, G. Dabbagh, L. N. Pfeiffer, and K. W. West, Science 268, 1460 (1995).

[13] G. J. Ferreira, H. J. P. Freire, and J. C. Egues, cond-mat/0607456

[14] R. Côté, A. H. MacDonald, Luis Brey, H. A. Fertig, S. M. Girvin, and H. T. C. Stoof,, Phys. Rev. Lett. 78, 4825 (1997); A. G. Green, Phys. Rev. B 61, 16299 (2000).

[15] M. Dobers, K. von Klitzing, J. Schneider, G. Weimann, and K. Ploog, Phys. Rev. Lett. 61, 1650 (1988).

[16] S. Kronmüller, W. Dietsche, J. Weis, and K. von Klitzing, Phys. Rev. Lett. 81, 2526 (1998).

[17] S. Kraus, O. Stern, J. G. S. Lok1, W. Dietsche1, K. von Klitzing, M. Bichler, D. Schuh, and W.Wegscheider, Phys. Rev. Lett. 89, 266801 (2002). 